This item was submitted to Loughborough's Research Repository by the author.

Items in Figshare are protected by copyright, with all rights reserved, unless otherwise indicated.

\title{
Release characteristics of polymer surface when moulding polyurethane
} foam

PLEASE CITE THE PUBLISHED VERSION

PUBLISHER

Maney Publishing on behalf of ( Institute of Materials, Minerals and Mining

LICENCE

CC BY-NC-ND 4.0

REPOSITORY RECORD

Majewski, Candice E., and Neil Hopkinson. 2019. "Release Characteristics of Polymer Surface When Moulding Polyurethane Foam". figshare. https://hdl.handle.net/2134/3501. 
This item was submitted to Loughborough's Institutional Repository by the author and is made available under the following Creative Commons Licence conditions.

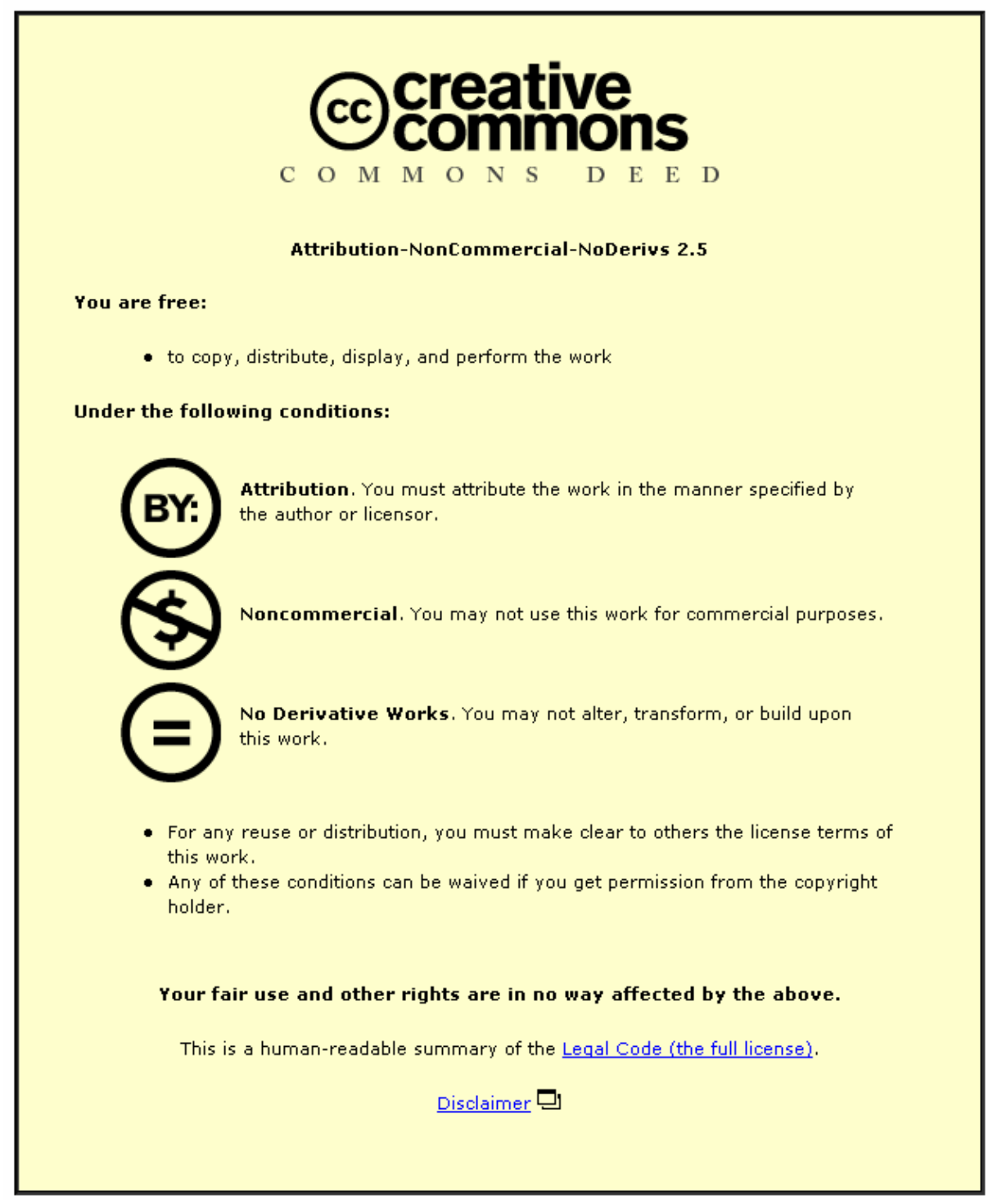

For the full text of this licence, please go to: http://creativecommons.org/licenses/by-nc-nd/2.5/ 


\section{Release characteristics of polymer surface when moulding polyurethane foam}

\section{E. Majewski* and N. Hopkinson}

The polyurethane (PU) foam moulding process involves the use of sacrificial release agents (SRAs) that are both costly and harmful to the environment. This research proposes the use of low surface energy, polymer substrates, as a means of eliminating SRAs from the foam moulding process.

Previous work identified the major factors affecting the ability of a polymer surface to release a $\mathrm{PU}$ foam part as being the surface energy and surface roughness of the substrate, and the proportion of isocyanate in the foam. The research described here has built upon these results and quantified the effects of each factor by using a $D$ optimal design of experiment structure. Crucially it has also been shown that, given the surface energy of a polymer substrate, its roughness and the composition of the foam, it is possible to predict whether or not unaided release should be possible, and a model has been produced in order to allow this prediction for the foams under consideration. This capability will provide the PU foam moulding industry with the possibility of identifying polymeric mould materials, and levels of finishing for these moulds, which have the potential to allow the elimination of SRAs from the production process.

Keywords: Polyurethane foam, Part release, Polymer mould, Surface energy

\section{Introduction}

\section{Polyurethanes}

The production of polyurethanes (PUs) relies on the fact that a polyisocyanate will react exothermically with a compound containing active hydrogen groups (for example a polyol) to produce the PU itself. ${ }^{1}$

Polyurethanes are one of the most versatile polymers, and can be produced in densities ranging from 6 to $122 \mathrm{~kg} \mathrm{~m}^{-3}$ and a wide array of stiffnesses. It is this versatility that has encouraged the use of PUs in their different forms throughout virtually any major industry that can be mentioned. Polyurethanes can be found in varying forms throughout almost every industry, whether as rigid insulation for refrigerators, as some form of adhesive, or as flexible foams for cushioning. One of the most common methods of forming a PU foam part is to take the two raw material components, meter them in a set ratio, and mix them just before injecting them into a mould. The mixture reacts exothermically within the mould, expanding to fill the tool.

\section{Part release}

The use of a traditional aluminium or steel mould generally requires the spray application of a mould release agent (MRA), normally before each moulding.

Wolfson School of Mechanical and Manufacturing Engineering, Loughborough University, UK

*Corresponding author, email c.e.majewski@lboro.ac.uk
Many of these spray-on MRAs emit volatile organic compound (VOC) emissions, which are the subject of European legislation $^{2}$ restricting the levels of these materials which may be released into the atmosphere. A previous study, carried out by the US Environmental Protection Agency, ${ }^{3}$ estimated VOC emissions from mould release agents to be $\sim 114000 \mathrm{t}_{\text {year }}{ }^{-1}$. The study also found that PU moulding operations accounted for $25 \%$ of the total MRA emissions, the majority of which were comprised of automobile seat and other foam moulding operations.

Additionally it has been shown that reducing or eliminating the use of release agents can achieve high productivity gains. ${ }^{4}$ One company alone ${ }^{5}$ currently spends $£ 150000$ annually on release agents, as well as owning $£ 275000$ of capital equipment which is specifically employed to apply these materials. In addition to these basic costs, substantial time is spent on mould cleaning to remove build-up of MRAs, costing between $£ 750$ and $£ 2500$ per tool per month.

A major disadvantage of using temporary release agents is the addition of an extra step to the moulding cycle. In the case of large tools, and depending on the method of application, this can account for fairly high percentages of the overall cycle. According to General Motors, the elimination of MRAs can save $25 \mathrm{~s}$ per part, and result in a $50 \%$ increase in productivity. ${ }^{6}$ It has ever been shown that it is possible to achieve increases in the number of parts produced per shift of $\sim 100 \%$ by the elimination of the release agent step of the cycle. $^{7}$ 
The limitations described here lead to the conclusion that the elimination of the requirement for MRAs would be extremely beneficial both to the PU foam moulding industry, and ultimately to the environment as a whole.

\section{Previous experimental results}

Previous research by the authors of the present work ${ }^{8,9}$ first confirmed the theory that a polymer mould could be used to provide unaided release of PU foam parts. However, it was also shown that not all polymers were capable of releasing the PU foam, and that some would only release under certain conditions. Three variables, the mould material (and more specifically its surface energy), the roughness of the polymer surface, and the proportion of isocyanate in the foam composition, were shown to be the dominant factors affecting the ability of the surface to release a PU foam part. Additionally, the temperature of the mould, and the demould time (the time between metering of the raw materials, and removal of the moulded part) were shown to have no significant effect on the likelihood of release. These findings have been built upon here, as discussed in the following sections.

\section{Experimental work}

\section{Aim}

The aim of the research reported here was to build upon the findings of the previous research first by quantifying the effects of each of the three factors identified as being significant on the ability of a polymer substrate to release a PU foam part. Second the present work sought to determine whether or not it would be possible to predict the likelihood of release given the input of the level of each of the factors. This ability, if shown to be possible, would have immense benefits for companies wishing to switch to a polymer mould, as it would demonstrate that the likelihood of release is not simply random, and rather that it is possible to assess whether or not a particular mould would be likely to release the specific raw material composition in use, before the requirement to produce costly tooling.

\section{Experimental methodology}

\section{Substrate selection}

Three materials, polytetrafluoroethylene (PTFE), polypropylene and polymethyl methacrylate (PMMA), were selected for testing, as the literature suggested that they would have a low, medium and high value of surface energy. ${ }^{10,11}$ Test substrates were produced from each of the three materials, in sheets of $150 \times 150 \times 5 \mathrm{~mm}$.

\section{Contact angle measurement and surface energy calculations}

The contact angles of two different liquids (diiodomethane and distilled water) were measured on each of three different substrate materials, using an OCA 20 machine from DataPhysics. ${ }^{12}$ The overall surface energy of each substrate was then calculated using equation (1), ${ }^{13}$ where $\gamma^{\mathrm{p}}$ is the polar component of the surface energy, and $\gamma^{\mathrm{d}}$ the dispersive component

$$
\frac{(1+\cos \theta) \gamma_{\mathrm{L}}}{2\left(\gamma_{\mathrm{L}}^{\mathrm{d}}\right)^{1 / 2}}=\left(\gamma_{\mathrm{S}}^{\mathrm{p}}\right)^{1 / 2}\left(\frac{\gamma_{\mathrm{L}}^{\mathrm{p}}}{\gamma_{\mathrm{L}}^{\mathrm{d}}}\right)^{1 / 2}+\left(\gamma_{\mathrm{S}}^{\mathrm{d}}\right)^{1 / 2}
$$

The use of diiodomethane, with no polar component, first allowed the dispersive component of the surface energy to be calculated. These values were then combined with the results obtained from distilled water, in order to calculate the polar component. The overall surface energy was then obtained simply by the addition of the two components. Three samples of each substrate material were tested, at four different positions on each. The average surface energy for each material can be seen in Table 1.

\section{Surface roughness preparation and measurement}

Once surface energy values had been obtained, the samples were roughened manually, and their $R_{\mathrm{a}}$ value measured using a Talysurf 4 surface profilometer from Taylor Hobson. ${ }^{14}$ Table 1 shows the values recorded for each level of roughness.

\section{Foam ratios}

Volumetric foam ratios were between a ratio of 10:7 (polyol/isocyanate) and 10:9. This range was selected to enable testing with a 10\% higher and $10 \%$ lower proportion of isocyanate than the $10: 8$ ratio most frequently used in production. Ratios were controlled using the gearing within the moulding equipment, and monitored regularly.

\section{Temperature and demould time}

Previous research ${ }^{9}$ had shown that the demould time and temperature did not have a significant effect on the likelihood of release. These were therefore not varied, and instead were maintained at constant levels throughout all experiments.

Table 1 summarises the levels selected for each factor.

\section{Experiment design}

A $D$ optimal design of experiment structure ${ }^{15,16}$ was used to generate a 40 run experiment, including low, medium and high $(-1,0,+1)$ levels of each of the three factors to be assessed, using the DesignExpert software available from StatEase Inc.

Flexible foam parts were produced using a polyol and isocyanate produced by Huntsman Polyurethanes, ${ }^{17}$ and moulded into a cylinder clamped onto the test substrate (see Fig. 1), in order to ensure that they were moulded onto the same area for each experiment. An M8 two component metering/mixing machine, manufactured by Unipre $\mathrm{GmbH}^{18}$ was used to ensure accurate and repeatable production of the foam. Parts were removed manually from the substrates, and the number of releases, up to a maximum of 10 parts, was recorded for each set of conditions.

Table 1 Experimental levels of factors

\begin{tabular}{|c|c|c|c|c|c|}
\hline Level & Surface roughness, $\mu \mathrm{m}$ & Surface energy, $\mathrm{mJ} \mathrm{m} \mathrm{m}^{-2}$ & Foam ratio (poly/iso) & Substrate temp., & Demould time, $\min$ \\
\hline-1 & $0 \cdot 1901$ & 18.4 (PTFE*) & $10: 7$ & Room temp. & 9 \\
\hline 0 & $0 \cdot 4205$ & $26 \cdot 6$ (polypropylene*) & $10: 8$ & Room temp. & 9 \\
\hline 1 & 0.6542 & 35.5 (PMMA) & $10: 9$ & Room temp. & 9 \\
\hline
\end{tabular}




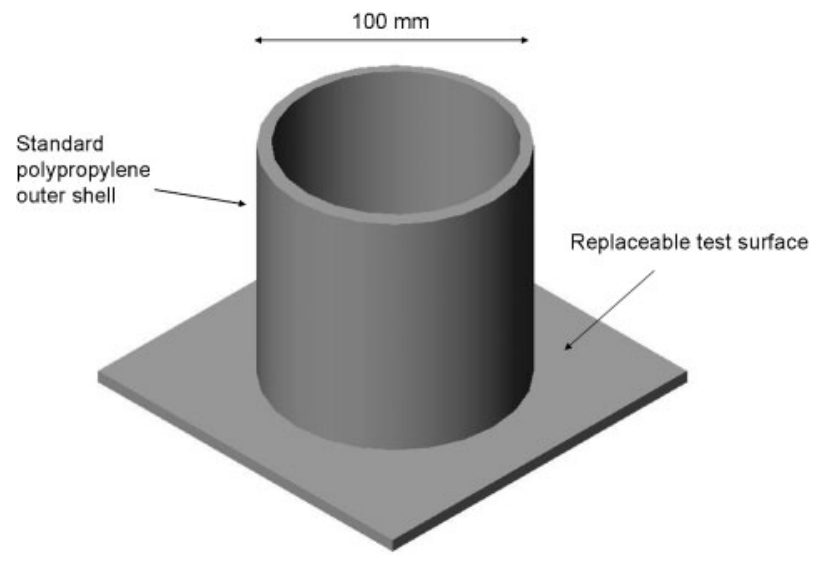

1 Mould geometry

\section{Experimental results}

As mentioned previously, each substrate was tested up to a maximum of 10 parts. Each experimental run in fact recorded a value of zero or ten releases, demonstrating that a substrate either will or will not release under a set of conditions, rather than perhaps releasing one or two parts and then failing. This is seen to be particularly important in the modelling work described at a later point.

\section{Significance of factors}

As discussed, the first aim of the present work was to quantify the effects of each of the three main factors, and to identify the hierarchy of importance for these factors. The following sections detail the effects of each of the main factors.

\section{Surface energy}

Figure 2 shows the average number of releases at each of the three original levels.

It can be easily observed that an increase in the surface energy of the substrate leads to a substantial decrease in the ability of that substrate to release the PU foam.

As discussed previously, the surface energy of a substrate will have a large influence on the formation of an adhesive bond with a moulded material. If a material in its liquid state cannot thoroughly wet the surface of the substrate, it is difficult for substantial adhesion to occur, as much of the substrate is not in intimate molecular contact with the moulded material.

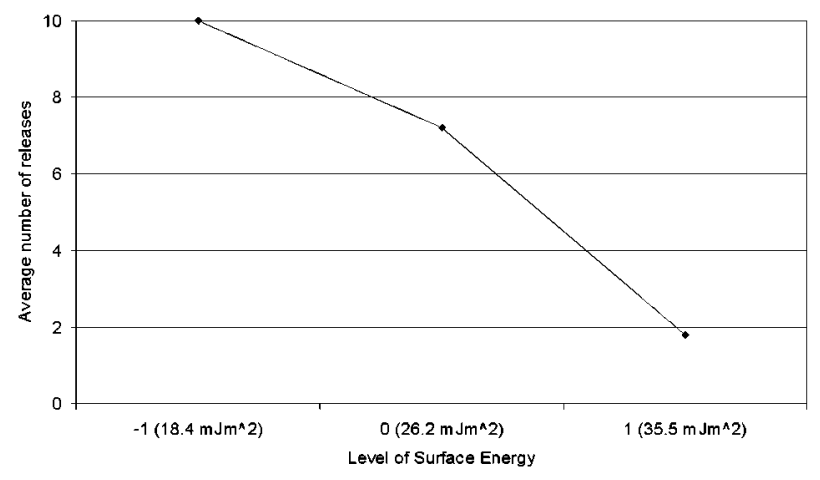

2 Average number of releases at each level of surface energy



3 Effect of surface roughness on average number of releases

However, in this case the lack of wetting achievable on a surface with a low surface energy is not the sole reason for the effect on the likelihood of release. In general the surface energy of a specific polymer can be related to the number of hydroxyl groups present in its structure. A substrate with a higher surface energy will generally have more hydroxyl groups than one with a lower surface energy. ${ }^{19}$ As discussed previously, the formation of a PU relies on the ability of an isocyanate to react with a material with a high concentration of active hydrogen. While the planned reaction in this process is with a polyol, the isocyanate will also react with any other hydroxyl compounds available, including those present in the polymer substrates being moulded onto here, forming chemical bonds across the interface between the part and its mould. The higher proportion of hydroxyl groups in the higher surface energy substrates therefore provides more opportunity for the formation of these chemical bonds, and therefore enables greater adhesion to take place.

\section{Surface roughness}

Figure 3 shows the effect of the surface roughness of the substrate on the average number of releases recorded.

It can be seen that an increase in the roughness of the surface led to a decrease in the likelihood of release. The use of a surface with a higher roughness provides a greater surface area for the moulded material over which adhesive bonds may form, resulting in an overall increase in adhesive force, and thus increased difficulty of part release.

\section{Foam composition}

Figure 4 shows the effect of the foam composition on the average number of releases.

It can be seen that an increase in the proportion of isocyanate in the mixture corresponded to a decrease in the ability to release a PU foam part from the substrate. As discussed in the results of the substrate surface energy, any excess isocyanate in the PU raw materials will be able to react with hydroxyl groups present on the surface of the substrate. As the proportion of isocyanate is increased, the amount available to form these bonds also increases, providing more chemical bonding across the interface, and therefore higher adhesive forces and more difficult release.

In order to show that the reason for the trend discussed in this section was in fact due to increased adhesion at higher proportions of isocyanate, rather than a reduction in the cohesive strength of the foam 


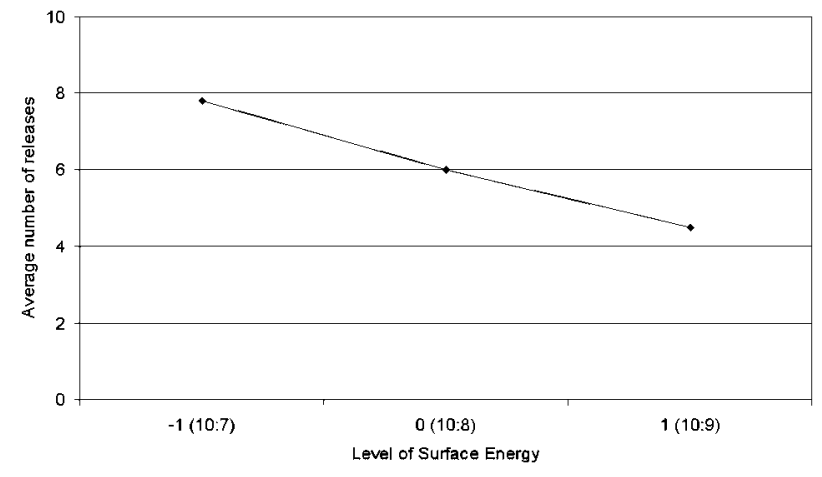

4 Effect of foam composition on average number of releases

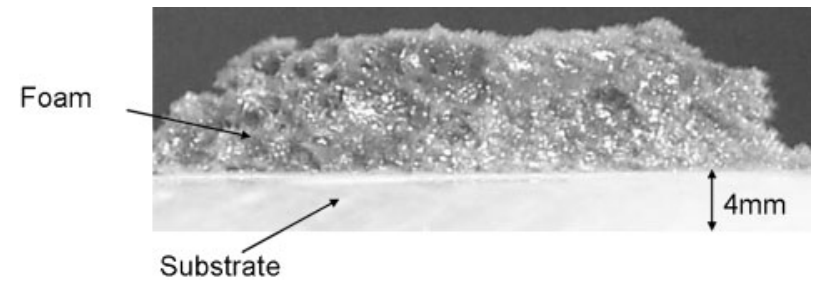

\section{Photograph of torn foam}

itself, in addition to analysing the results gained from varying the foam composition, tensile tests were carried out on the foam at each of the three main ratios. Figure 5 shows a photograph of a part which failed to release. It can be seen that, rather than failure occurring at the interface between the moulded foam and the substrate, tearing occurred within the foam itself. This led to a proportion of the foam remaining stuck to the substrate, as shown in the photograph.

The tensile tests were therefore carried out, using an Instron 3366 materials testing system. ${ }^{20}$ Figure 6 shows the tensile strengths of a series of parts produced at each ratio.

It can be seen that the tensile strength of the foam actually increased with increasing proportions of isocyanate, thereby confirming that the foam was not weaker at these high ratios, and that it could confidently be stated that the increased difficulty of release was in fact related to the adhesive forces developed over the interface.

\section{Summary of main factor effects}

Table 2 summarises the main factors and the effect on the average number of releases for each.

It can be seen that the greatest effect was observed from changing the surface energy of the substrate, followed by its surface roughness. The foam composition, while also having an effect, was the least important of the three factors.

\section{Interactions}

In addition to assessing the effects of the main factors, it is also relevant to include a brief discussion of the

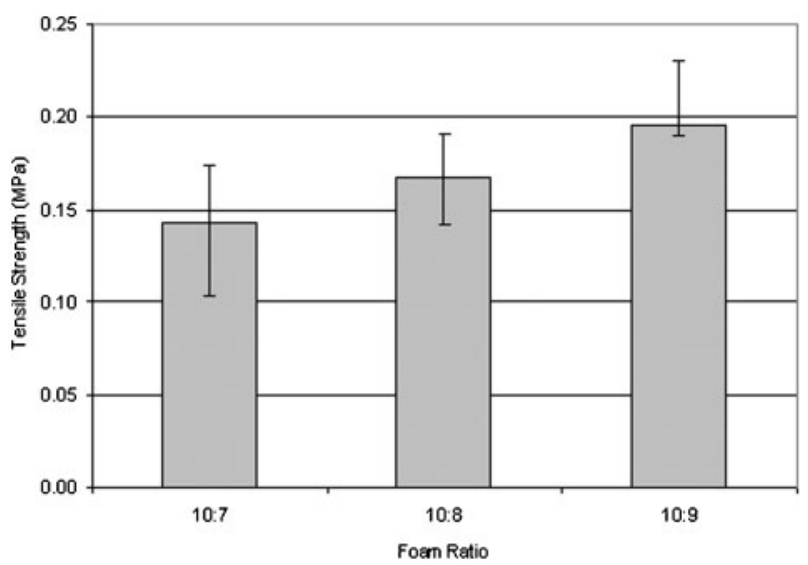

6 Tensile strength at each ratio

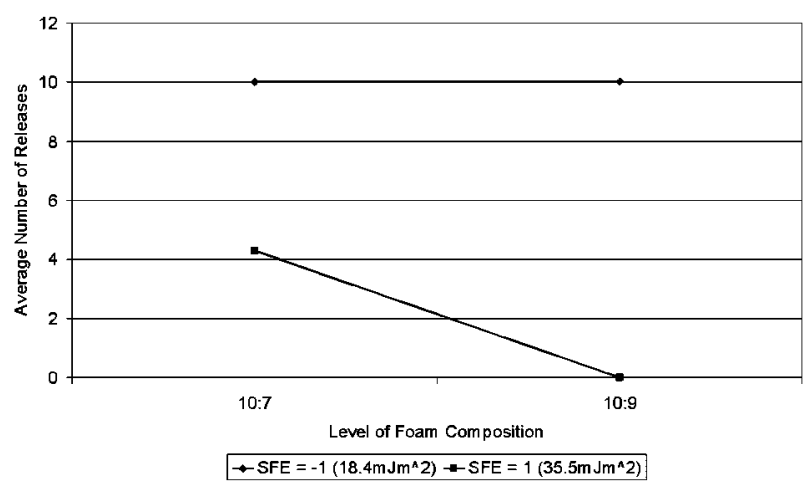

7 Interaction between surface energy and foam composition

interactive effects between these factors. It is often the case in any process that the effect of changing the level of one factor is greater at a high level of another factor than that at the lower level, as will be seen in the following discussions.

\section{Surface energy/foam composition}

Figure 7 shows the effect of the level of surface energy on varying the level of foam composition.

It can be seen that at the low value of surface energy the foam composition showed no effect on the number of releases achieved, whereas at the high level of SFE an increase in the isocyanate content of the foam corresponded to a decrease in the likelihood of release. As discussed previously, at a high level of surface energy there is a higher number of hydroxyl compounds available to react with excess isocyanate in the mixture, leading to a further increase in adhesion as the proportion of isocyanate is increased. However, when considering a substrate with a very low surface energy, there are so few reactive groups present that even when an excess of isocyanate is present there will still be extremely limited possibilities for chemical bonding.

Table 2 Effect of each factor

\begin{tabular}{lccc}
\hline Factor & Average (low level) & Average (high level) & Difference \\
\hline Surface energy & 10 & $1 \cdot 8$ & $-8 \cdot 2$ \\
Surface roughness & $8 \cdot 2$ & 4.5 & $-3 \cdot 7$ \\
Foam composition & $7 \cdot 8$ & 4.5 & $-3 \cdot 1$ \\
\hline
\end{tabular}




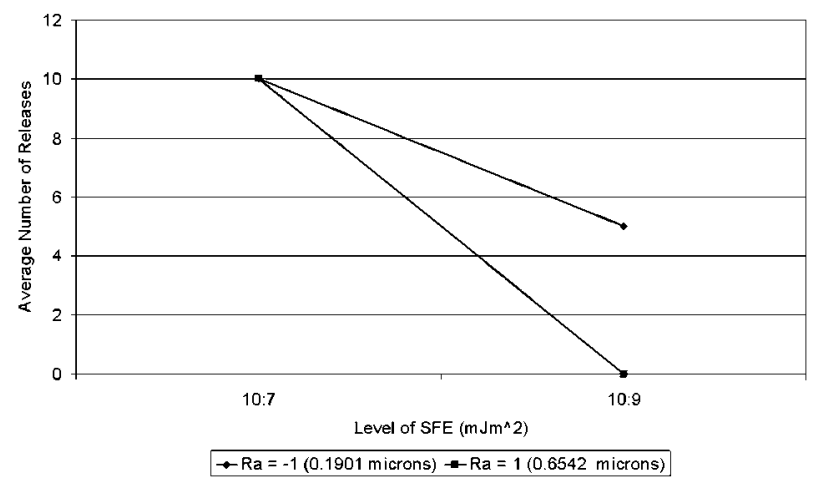

8 Interaction between surface roughness and surface energy

\section{Surface roughness/surface energy}

Figure 8 shows the effect of different levels of roughness on varying the surface energy of the substrate.

It can be seen that at a low level of roughness, the effect of increasing the surface energy is less than that at higher roughness levels.

The manufacturing method for the mould, and the level of finishing, would be expected to have an effect on the roughness of the surface; however, a higher level of finishing would often add cost and time to the production of the mould. A manufacturer would need to take these factors into consideration before embarking on production of a mould, and would need to determine whether it would be more cost effective to use a rougher mould manufactured from a material with a lower surface energy material, or to produce a mould from a different material, but with a higher level of finishing.

\section{Surface roughness/foam composition}

Figure 9 shows the interaction between the surface roughness and the foam composition.

It can be seen that the level of surface roughness had very little effect on the effect of the foam composition on the likelihood of release, and is clearly the least significant of the interactions discussed here.

It can clearly be seen from the preceding discussions that, when considering a substrate with a low surface energy, the release process is substantially more robust to variations in the remaining two factors. This suggests that fluctuations in the proportion of isocyanate, or in the roughness of the surface, would have less of a detrimental effect when using a substrate such as PTFE. However, the cost of producing a PTFE mould may well be greater than

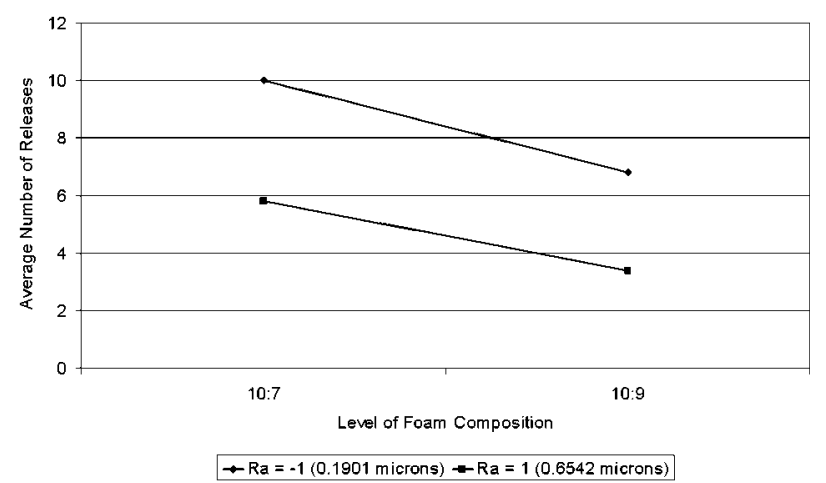

9 Interaction between surface roughness and foam composition

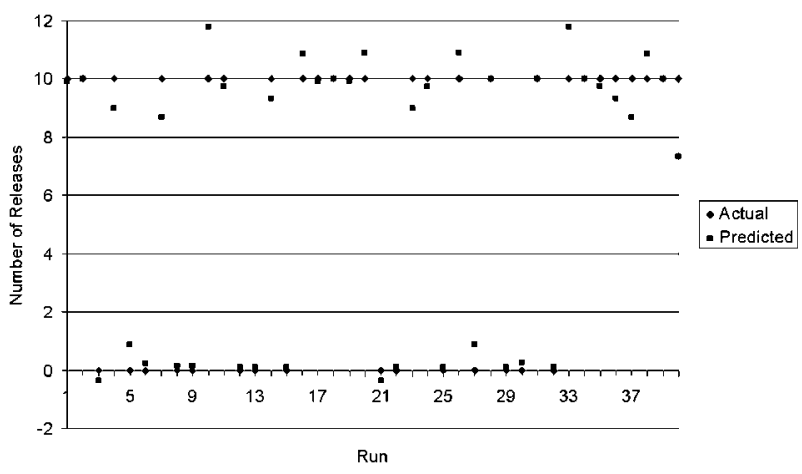

10 Actual and predicted number of releases

that for other polymer with slightly higher surface energies. In this case, the ability to predict the likelihood of release under certain conditions would be invaluable to a manufacturer wishing to produce a mould which is capable of providing unaided part release, while at the same time minimising the costs of this production.

\section{Prediction of release}

The results of the experimental work were analysed using the DesignExpert software and used to create a model to predict the likelihood of release (equation (2)). As the experimental work recorded a numerical value of the releases, it was not possible to generate an exact 'yes or no' response, but it was anticipated that a range of predicted values could be established within which it could be confidently stated that release or non-release would occur

$$
\begin{aligned}
R= & 7 \cdot 07-2 \cdot 45 A-5 \cdot 73 B-2 \cdot 43 C+1 \cdot 23 A^{2}- \\
& 3 \cdot 43 B^{2}+1 \cdot 20 C^{2}-1 \cdot 15 A B+1 \cdot 00 A C-1 \cdot 14 B C+ \\
& 1 \cdot 03 A^{2} B-2 \cdot 47 A^{2} C+3 \cdot 53 A B^{2}-2 \cdot 46 A C^{2}+ \\
& 3 \cdot 53 B^{2} C+1.03 B C^{2}+1 \cdot 28 A B C
\end{aligned}
$$

Figure 10 shows the actual and predicted values for each of the 40 experimental runs. It is important to note that the 'actual' values presented here do not signify that a substrate would only release 10 parts before failing, as the maximum number of parts tested was 10 . A substrate returning a value of zero released would therefore be classed as showing no potential to be self-releasing, and one recording the full ten releases would be considered to be a viable option to provide unaided part release.

It can be seen that all but one data point (run 40) was predicted within \pm 2 of the actual number of releases, and that there was a definite range of values (between 2 and 8 ) within which no points were predicted. It was therefore considered that these ranges could be classed as a benchmark for predicting the likelihood of release. Table 3 shows the range of values within which release or non-release could be predicted with some confidence.

\section{Validation of model}

In order to verify and improve upon the model discussed in this section, a selection of further experiments was performed, and the accuracy of the predictions were assessed. A random selection of 10 additional experimental runs was made, featuring at least one intermediate level not previously assessed of one of the factors. It was not possible to obtain a readily available engineering 




11 Predicted values for validation runs

material at the lower intermediate level of surface energy, so only one additional level of this factor, polyethylene $(0.5$ level $)$, was included. Table 4 shows the revised factor levels available for selection. As previously, the demould time was maintained at $9 \mathrm{~min}$, and the mould was held at room temperature throughout.

Figure 11 shows the actual and predicted values for each of the ten validation runs carried out.

It can be seen that in some cases the predicted values were less accurate for these intermediate points than for the previous experimental points, although the predicted values for all samples not releasing fell below 4, and all those for samples which did release were $>7$. However, it was felt that the use of the results gained here would allow the overall refinement of the model.

A refined model was produced (equation (3)), taking into account the results from the intermediate points used for validation

$$
\begin{aligned}
R= & 7 \cdot 10-8 \cdot 23 A-13 \cdot 70 B-2 \cdot 53 C+1 \cdot 30 A^{2}- \\
& 2 \cdot 44 B^{2}+0 \cdot 24 C^{2}-0 \cdot 98 A B+0 \cdot 90 A C- \\
& 1 \cdot 13 B C+6 \cdot 14 A^{3}+9 \cdot 64 B^{3}-1 \cdot 98 A^{2} C+ \\
& 2 \cdot 66 A B^{2}-1 \cdot 98 A C^{2}+3 \cdot 21 B^{2} C+1 \cdot 21 A B C
\end{aligned}
$$

Figure 12 shows the predictions provided by the revised model; the results of the original model are included for comparison.

It can be seen that the accuracy of predictions for these points was substantially greater than those gained when using the original model. The average deviation (residual) from the actual results was 1.97 for the original model prediction; this deviation was reduced to 1.14 in the revised model. Figure 13 now shows the predictions from

Table 3 Range of values for prediction

\begin{tabular}{ll}
\hline Predicted value & Expected release \\
\hline$(-2)-(2)$ & No \\
$(2)-(8)$ & Inconclusive \\
$(8)-(12)$ & Yes \\
\hline
\end{tabular}

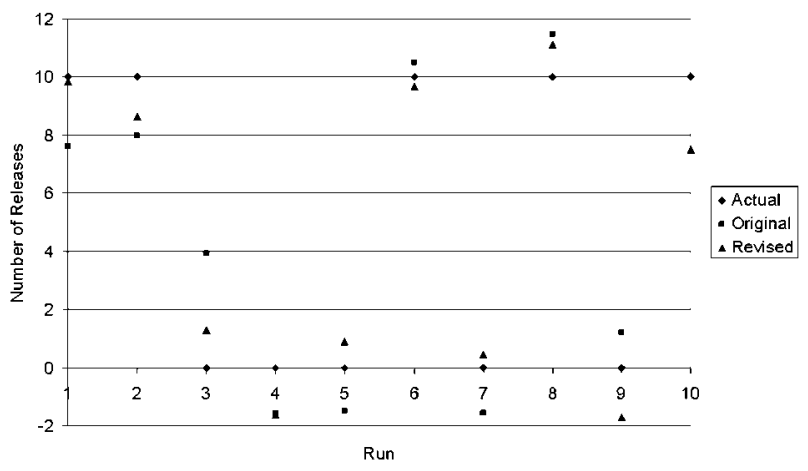

12 Predicted values (original and revised models)

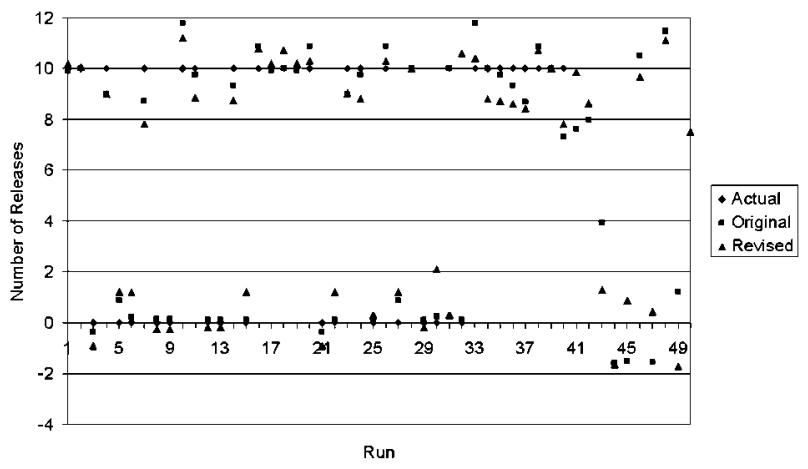

13 Predicted results from both models

both models for the whole data set, including all 40 original runs, and the further 10 included for validation.

The mean residual for the revised model was slightly higher than that for the original model $(0 \cdot 89$ versus 0.81 ); however, the standard deviation of the data set was lower $(0.63$ versus $0 \cdot 91)$. Although there was some perceived difference in the accuracy of the two models, particularly for the validation runs, a $T$ test carried out on averages of the two data sets showed that, overall, there was no statistical difference between the two. It was therefore decided that any further experimentation and model refinement would not be expected to show a substantial improvement, and therefore no further work was carried out on this.

\section{Conclusions}

The ability to eliminate the use of release agents has many potential implications, both for PU foam manufacturers and for the wider community in general. However, the release of PU foam parts is currently somewhat of a 'black art', with manufacturers relying on trial and error to find a suitable solution, and leading to a reluctance by many to investigate new solutions. This research has attempted to show that a more scientific approach can be taken to the problem, and that it is possible to identify certain mould parameters and

Table 4 Revised factor levels

\begin{tabular}{llll}
\hline Level & Surface roughness $(\boldsymbol{A}), \boldsymbol{\mu m}$ & Surface energy $(\boldsymbol{B}), \mathrm{mJ} \mathrm{m}^{-2}$ & Foam ratio $(\boldsymbol{C})$ (polyol/iso) \\
\hline-1 & 0.1901 & $18 \cdot 4$ (PTFE) & $10: 7$ \\
-0.5 & 0.3086 & N/A & $10: 7 \cdot 5$ \\
0 & 0.4205 & $26 \cdot 6$ (polypropylene) & $10: 8$ \\
0.5 & 0.5316 & $30 \cdot 84$ (polyethylene) & $10: 9$ \\
1 & 0.6542 & $35 \cdot 5$ (PMMA) & 10 \\
\hline
\end{tabular}


operating conditions under which unaided part release is most likely.

The research described here has shown that it is possible to predict, with a relatively high degree of accuracy, the likelihood of releasing a PU foam part from a polymer substrate, given the input of the surface energy and roughness of the substrate, and the composition of the foam itself. This in turn will allow a foam manufacturer to identify a range of mould parameters under which release could be expected, preventing the costs and times associated with attempting to test a wide range of suitable materials.

It has also been shown that the surface energy of a polymer mould will have the most dominant effect on the release of PU foam moulded parts, followed by the roughness of the mould itself, and finally the proportion of isocyanate in the foam.

This confirms that the surface energy of the mould is the major determining factor in whether or not part release can be expected, and also that at low surface energies the process is most robust to changes in other parameters. Coupled with the less major effect of the foam composition, this has promising implications given the fluctuations in foam composition that occur during the normal running of the moulding process.

The effect of surface roughness suggests that if even further enhanced release is required it would also be possible to increase the level of finishing applied to the mould in order to lower the roughness and improve the chances of release. This may also in some cases affect the choice of manufacturing method for the mould itself.

\section{References}

1. Bayer: 'Polyurethanes', technical handbook, 1979.

2. 'The limitation of emissions of volatile organic compounds due to the use of organic solvents in certain activities and installations',
Council Directive 1999/13/EC, Official Journal L 085, March 29, 1999.

3. J. Lanning and K. Cavender: 'Evaluation of pollution prevention opportunities for mold release agents', United States Environmental Protection Agency Project Summary, July 1996.

4. W. Willkomm, R. Jennings and C. Macosko: Plast. Rubber Compos. Process. Appl., 1993, 19, 69-76.

5. Collins \& Aikman Automotive Interior Systems Europe. St. Neots, UK, available at www.collinsaikman.com

6. J. Sneller: Modern Plast., 1984, 6, (7), 66-68.

7. M. Cekoric, R. Taylor and C. Barrickman: 'Internal mold release the next step forward in RIM productivity', SAE Technical Paper Series, Detroit, MI, 1983.

8. N. Hopkinson: 'Laminate tooling for polyurethane foam moulding', IMCRC Project \#41, Loughborough University, 2001-2004.

9. C. E. Majewski and N. Hopkinson: Proc. Polyurethanes Industry Polyurethanes Technical Conf., Las Vegas, NV, USA, October 2004, 377-385, The Alliance for the Polyurethane Industry.

10. D. Packham: 'Handbook of adhesion', (ed. D. Packham), 455-458; 1992, Harlow, Longman Scientific and Technical.

11. D. van Krevelen: 'Properties of polymers: correlations with chemical structure'; 1972, Amsterdam, Elsevier.

12. DataPhysics Instruments GmbH Filderstadt, Germany, available at www.dataphysics.de/

13. D. Owens and R. Wendt: J. Appl. Polym. Sci., 1969, 13, 17411747.

14. Taylor Hobson Ltd Leicester, England, available at www. taylor-hobson.com

15. R. Fisher: 'Design of experiments', 8th edn; 1966, London, Oliver and Boyd.

16. R. Launsby: 'Straight talk on designing experiments: an introductory design of experiments reference handbook', Launsby Consulting, Colorado Springs, 1993.

17. Huntsman Polyurethanes, available at www.huntsman.com/pu/

18. Unipre GmbH Werl, Germany, available at www.unipre.de/

19. Institute of Polymer Technology and Materials Engineering (IPTME), Loughborough University, UK, available at www.lboro. ac.uk/departments/iptme/index.html

20. Instron Limited, European Headquarters High Wycombe, UK, available at www.instron.co.uk 